\title{
Current Trends in Ophthalmology Patients Preference of Diquafosol or Rebamipide Eye Drops for the Treatment of Mild Dry Eye: A Pilot Study
}

\author{
Maria Ohtani ${ }^{1}$, Yukihiro Horie ${ }^{1}$, Yoshiaki Tagawa ${ }^{2}$, \\ Susumu Ishida ${ }^{2}$ and Nobuyoshi Kitaichi ${ }^{1,2}$
}

\author{
1Department of Ophthalmology, Health Sciences University of Hokkaido, Sapporo, Japan \\ 2Department of Ophthalmology, Hokkaido University Graduate School of Medicine, Sapporo, Japan
}

\begin{abstract}
*Correspondence: Nobuyoshi Kitaichi, Ainosato 2-5, Kita-ku, Sapporo 002-8072, Japan, Tel: +81-11-778-7575; Fax: +81-11-770-5034; E-mail: nobukita@hoku-iryo-u.ac.jp
\end{abstract}

Received: May 17, 2018; Accepted: July 09, 2018; Published: July 11, 2018

\begin{abstract}
Purpose: To investigate the preference rates of recently developed eye drops, 3\% diquafosol ophthalmic solution and $2 \%$ rebamipide ophthalmic suspension, as a randomized clinical trial in patients with dry eye.

Methods: Eighteen patients (mean age: 59.7 years old, females: 77.8\%) with reduced tear film break-up time (TBUT $\leq 5 \mathrm{sec}$ ) were enrolled in this study. They were treated with either diquafosol or rebamipide for 1 week, and treated for an additional week after switching to the other eye drop. This treatment was repeated one more time. Then, the patients were treated with one of either eye drop for an additional 4 weeks. Patients' preferences for eye drops were investigated using a questionnaire at the end of 4-week treatment. To confirm the effectiveness of the treatments, objective and subjective assessments including superficial punctate keratopathy (SPK) score, TBUT measurement, and Dry Eye-Related Quality-of-Life Score (DEQS) were performed at baseline, and 4 and 8 weeks.

Results: SPK score was improved after treatment $(P<0.05)$. Patients tended to prefer diquafosol $(64.7 \%)$ to rebamipide (35.4\%), however there was no significant difference in two eye drops $(P=0.23)$. Bitterness was reported significantly more frequently after rebamipide administration (66.7\%) than diquafosol administration $(5.6 \%, P<$ $0.001)$. The diquafosol bottle was considered "ease to use" by a larger percentage of users (94.1\%) as compared to the rebamipide bottle $(58.8 \%, P<0.05)$. The effectiveness of the signs and symptoms were similar for both groups at 8 weeks.
\end{abstract}

Conclusions: Diquafosol and Rebamipide were both effective in treating dry eye and 65\% of the patients choose Diquafosol. Bitterness after administration and ease to use of the bottles seemed to affect the patients' preferences for these two eye drops.

Keywords: Dry eye; Diquafosol; Rebamipide

\section{Introduction}

Dry eye is a multifactorial disease of the tear film and ocular surface that results in symptoms of discomfort, visual disturbance and tears film instability with potential damage to the ocular surface [1]. It has a high prevalence worldwide, with the reported incidence rates varying from approximately $5 \%$ to $35 \%$, depending on the population studied [2]. Older age and female sex are widely recognized as the two most common risk factors for dry eye, based on the results of multiple epidemiological studies [2-5]. The pathophysiology of dry eye includes two mutually reinforcing mechanisms; the vicious circle between tear film instability and the ocular surface epithelial damage [6]. Keratoconjunctival epithelial damage and tear film instability eventually lead to aggravation of dry eye, and also to subjective symptoms, such as eye discomfort, 
foreign body sensation or visual disturbance [7], thereby lowering the patient's Quality of Life (QOL) [8] or Quality of Vision (QOV) [9].

Currently, artificial tears, sodium hyaluronate solutions, corticosteroids and cyclosporine were utilized as conventional therapies for dry eye [10-12], however, these therapies have limited efficacy and safety. "Artificial tears" refers to temporary water and electrolyte supplementation [13]. Sodium hyaluronate solution has shown some effectiveness in patients with dry eye [14-16], however it is considered ineffective against conjunctival disorders caused by mucin layer damage [14]. Corticosteroids and cyclosporine should be avoided in some subjects for reasons like increased intraocular pressure, cataract, infection, and insurance system in Japan.

Recently, two pharmacological agents Diquafosol [17], an agonist of the $\mathrm{P} 2 \mathrm{Y}_{2}$ receptor involved in water and mucin secretion, and Rebamipide [18] which can increase corneal and conjunctival mucin-like substances, were launched in Japan. Diquafosol ophthalmic solution is reported to significantly improve objective markers of dry eye disease such as corneal and conjunctival fluorescein staining in several randomized controlled trials, and improve tear film break-up time and Schirmer test scores in some studies [19-21]. It was reported that diquafosol sodium improved Schirimer test value and mucin-like substances among patients with dry eye [22,23]. Now diquafosol eye drops can be prescribed in China and Korea for patients with dry eye following Japan.

Rebamipide was initially developed and approved for use in treating gastric ulcers and lesions associated with gastritis. For the ophthalmic application, rebamipide ophthalmic suspension can effectively treat tear deficiency and mucin-related corneal epithelial damage, and restore the microstructure responsible for tear stability [18]. It was reported that the mucinlike substance on conjunctiva and cornea was increased by using Rebamipide in rabbit model [24]. Conjunctival goblet cell density significantly increased in patients with dry eye by using rebamipide [25]. Due to these favorable data, diquafosol and rebamipide are now widely used options for dry eye treatment in Japan.

To accumulate the evidences and to identify the eligible way of using these agents are very important clinically. In clinical practice, patient preference is of great significance because it strongly affects the treatment adherence of patients with dry eye who require long-term therapy. Therefore, we conducted a survey to investigate the preferences of patients with dry eye with respect to specific eye drops, diquafosol ophthalmic solution and rebamipide ophthalmic suspension, in a clinical study.

\section{Materials and Methods Study Design}

This is a prospective, randomized controlled crossover study. Envelope method was approved as a sampling technique. Subjective and objective findings were assessed by the different observers. This study consisted of a survey to investigate patient preferences with respect to $3 \%$ diquafosol ophthalmic solution (Diquas ophthalmic solution $3 \%{ }^{\circledR}$, Santen Pharmaceutical Co., Ltd, Osaka, Japan) and $2 \%$ rebamipide ophthalmic suspension (Mucosta ophthalmic suspension UD2\% ${ }^{\circledR}$, Otsuka Pharmaceutical Co., Ltd, Tokyo, Japan) for the treatment of dry eye. After a screening period, patients were treated with either diquafosol or rebamipide for 1 week, and treated for an additional week after switching to the other eye drop. This treatment cycle was repeated one more time, and then patient preferences of eye drops were investigated with a questionnaire given at the end of the 4-week treatment. The patients selected their favorite one of two, and were treated with it for an additional 4 weeks. The patients were instructed to place a drop into the eye 4 times daily. The effectiveness of the eye drops was also confirmed at week 4 and 8 (Figure 1).

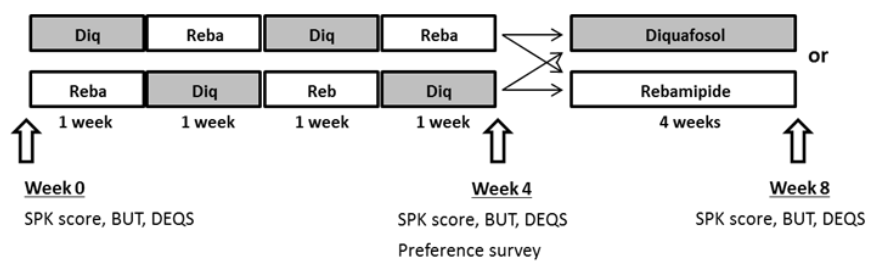

Figure 1: Study design. SPK; superficial punctate keratopathy, TBUT; tear film break-up time, DEQS; Dry

Eye-Related Quality-of-Life Score.

Institutional Review Board of the Institute for Personalized Medicine, Health Sciences University of Hokkaido approved the protocol before the study initiation (No. 2013-001). This research was conducted in compliance with the Declaration of Helsinki, and prior written informed consent was obtained from all study patients.

\section{Patients}

The study subjects were adult patients (aged 20 years old or older) who complained dry eye-related symptoms, and visited the Department of Ophthalmology, Health 
Sciences University of Hokkaido Hospital, between June and October 2013, and were subsequently diagnosed as having dry eye disease according to the dry eye diagnostic criteria defined in 2006 by the Japanese Dry Eye Society [26]. Fluorescein paper was used to evaluate BUT and SPK. Corneal fluorescein staining was scored based on the modified criteria established by von Bijsterveld; each eye is divided into 3 sections (temporal conjunctiva, cornea and nasal conjunctiva) and scored from 0 to 3 ( 0 , negative; 1 , scattered minute; 2 , moderate spotty; 3 , diffuse blotchy staining). Patients with poorly-controlled diabetes, severe allergic conjunctivitis, significant conjunctival chalasis, and serious epithelial or endothelial damages were excluded from this study. No other subjects were participated as mild dry eye cases.

\section{Study Assessments}

Patient preferences for eye drops were investigated with a questionnaire at the end of 4-week treatment cycle. The questionnaires included "Which ophthalmic solution do you prefer?" and questions asking impression of each eye drop after use such as "Comfortable", "Moistened", "Be better" or "See clearly", and feeling after use such as "Nothing", "Irritation", "Bitterness" or "Discharge", and evaluation of each eye drop's bottle such as "Easy to use".

Objective assessment using SPK score or TBUT, and subjective assessment using Japanese version of DEQS [27] were also performed to confirm the effectiveness at baseline, week 4 and 8.

\section{Statistical Analyses}

For continuous variables, descriptive statistics included the number of patients $(\mathrm{N})$, mean and standard deviation (SD). For categorical variables, the number and percentage of patients were calculated. Patient preferences derived from responses to "Which ophthalmic solution do you prefer?" were analyzed with chi-square test and the responses to impressions and feelings following each eye drop application, and evaluations of each eye drop's bottle were analyzed with McNemar's test. For efficacy evaluations, the changes from baseline variables at week 4 were analyzed using Wilcoxon signed-rank test and pairwise comparisons between two eye drops at week 8 was analyzed using Wilcoxon rank-sum test (TBUT and DEQS). Similarly, the changes from baseline variables were analyzed using a paired t-test, and pair-wise comparison was analyzed using the Student's t-test (SPK score). The significance level was set at 0.05 using two-sided tests.

\section{Results}

\section{Demographics and Baseline Characteristics}

Eighteen dry eye patients (mean age: 59.7 years old, 14 female: $77.8 \%$ ) were enrolled in this study. They included 1 dentist, 1 nurse, 1 librarian, 1 office worker, 2 drivers, and 12 retired persons/housekeepers. The SPK score, TBUT and DEQS at baseline was $2.5 \pm 2.2$ (mean \pm SD), 2.6 $\pm 1.2 \mathrm{sec}$. and $34.6 \pm 28.0$, respectively.

\section{Patient Eye Drop preferences}

Among the 18 patients, 17 patients answered the question related to their preferred eye drop among diquafosol and rebamipide (response rate: $94.4 \%$ ) (Figure 2). Diquafosol (11 patients, 64.7\%) tended to be preferred to rebamipide (6 patients, 35.3\%), however, there was no statistical significance between two eye drops $(P=0.23$, chi-square test).

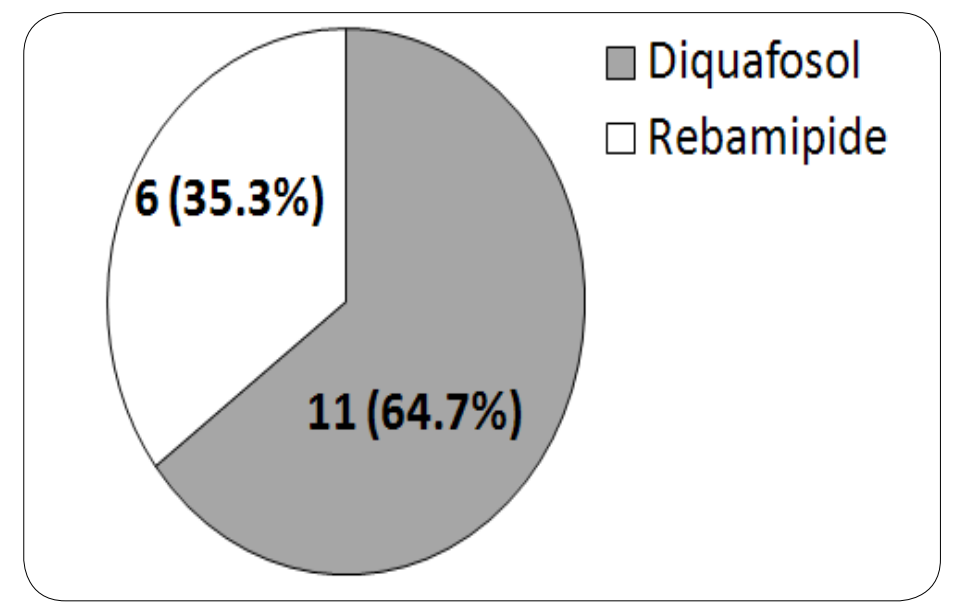

Figure 2: Questionnaire investigating the preferred eye drops among Diquafosol and Rebamipide. Patients answered the question 'what ophthalmic solution do you prefer?' P value was 0.225 (chi-square test).

In the questionnaire survey, patients' impressions and feelings of each eye drop after use, and patients' evaluations of eye drop bottles were also investigated. Bitterness was reported significantly more often with rebamipide (12 patients, 66.7\%) than diquafosol (1 patients, $5.6 \%, P<0.001)$. A larger fraction of patients answered that the diquafosol bottle was easier to use (17 patients, 94.1\%) as compared to the rebamipide bottle (10 patients, 58.8\%, $P<0.05$ ). No significant differences were observed for other questionnaire items related to patient impressions, such as "comfortable", "moistened", "be better", "see clearly" or feelings after administration. Patients who answered that "the bottle is harder to use" for rebamipide were older (mean age \pm SD; $65.3 \pm 6.3$ years old) than patients who answered "easy to use" 
(mean age $\pm \mathrm{SD} ; 54.8 \pm 7.6, P<0.01$ ) (Figure 3 )

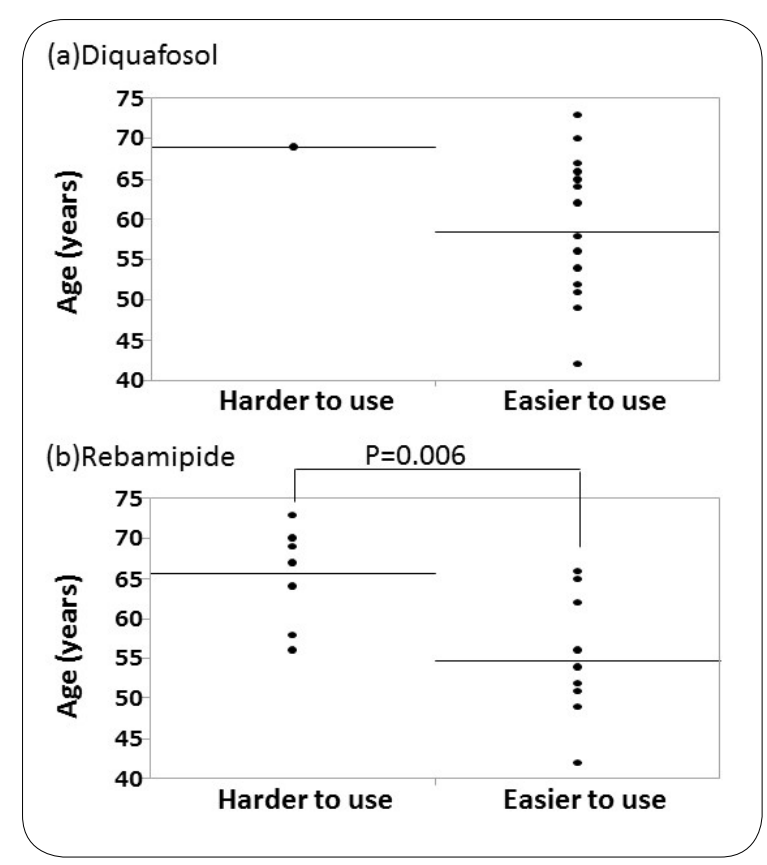

Figure 3: Sub-analyses for the evaluations of each eye drop's bottle based on patient's ages. The age of patients (Total $\mathrm{N}=17$ ) who answered "The bottle is harder to use" or "easier to use" for (a) diquafosol and (b) rebamipide are plotted, and compared for each eye drop. $P$ value was calculated by student's t-test.

\section{Effectiveness}

SPK scores significantly improved after 4 weeks of treatment with diquafosol and rebamipide compared to baseline (Figure $4 a, P<0.05$ ). No significant difference in SPK score was observed at week 8 between the two groups (Figure 4a). TBUT tended to be prolonged at week 4 compared with baseline, but this was not significant, and the effectiveness of the two eye drops was similar at the end (Figure 4b). The patients' QOL subjectively assessed using DEQS, also tended to be improved (Figure $4 c)$.

\section{Discussion}

Dry eye is recognized as a chronic disease, frequently requiring long-term management [11]. As with many therapies for chronic diseases, some patients with dry eye do not adhere to long-term treatment with eye drops. To improve adherence to dry eye therapies, patient education, including direct instructions from physicians as well as an additional discussion with clinical staff focusing on duration of therapy, potential ocular adverse effects and tolerability, is thought to be an essential for the initiation of repeated courses of topical application of eye drops [28].

\section{(a) SPK scores}

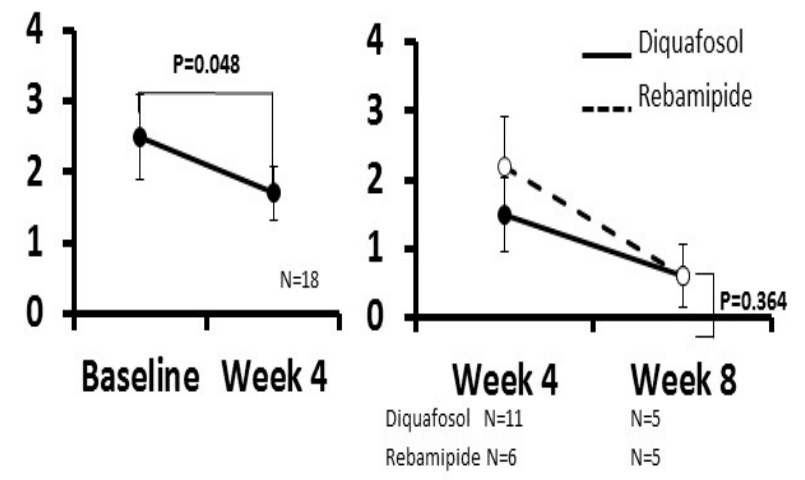

(b) BUT (sec)

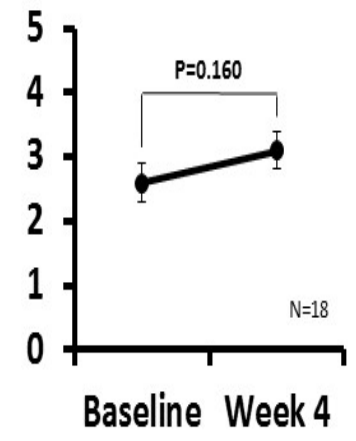

(c) DEQS
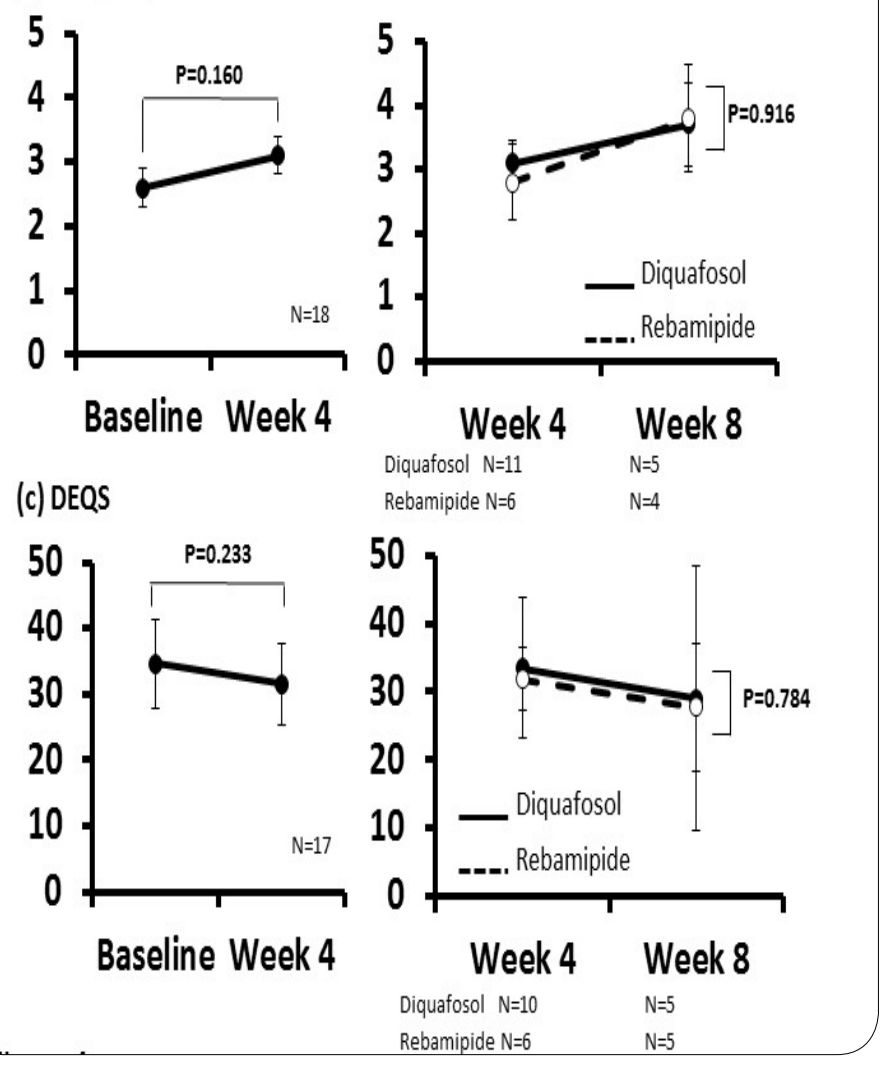

Figure 4: Efficacy variables of SPK score, TBUT and DEQS.

Each parameter was compared with baseline at week 4, and between groups at week 8. (a) SPK and (b) DEQ scores were analyzed by Wilcoxon signed-rank test in comparing baseline to at week 4 , and by Wilcoxon rank-sum test in comparing between groups at week 8. Similarly, the change in (c) TBUT from baseline value was analyzed at week 4 by paired t-test, and compared between groups at week 8 by student's t-test.

Diquafosol is now widely used in Japan, China, and Korea. These 2 new eye drops would be approved for clinical use in other Asian countries before too long. Our present results provide useful information 
to ophthalmologists and patients in other upcoming regions.

There was no statistical significance in preference between diquafosol and rebamipide, but some impression of the patients was different. The results of this survey revealed that bitterness was reported significantly more often with rebamipide than with diquafosol, and more patients found the bottle of diquafosol easier to use than that of rebamipide. These results seemed to correlate with the ratio of patients' preferences with respect to these eye drops.

Rebamipide was reported to be effective and welltolerated in a randomized multicenter phase III study, however dysgeusia (bitter taste) was commonly observed as an adverse event [28]. This is consistent with our survey results in clinical practices. As for diquafosol, eye irritation occurred with the highest frequency in a randomized multicenter phase 3 studies [21]. The results of the present survey also confirm the clinical trial data, indicating that irritation was reported more frequently with diquafosol than with rebamipide, but with no significant difference.

Diquafosol ophthalmic solution and rebamipide ophthalmic suspension differ with respect to the clinically supplied bottle-type, as the former is contained in multidose bottles containing $5 \mathrm{~mL}$ of solution each, while the latter is contained in disposable single-dose bottles containing $0.35 \mathrm{~mL}$ of suspension without preservatives. We conclude that a multi-dose bottle is more convenient for 4 to 6 time's daily administration, and this is why more patients preferred diquafosol. Furthermore, the aged patients who responded "hard to use" was significantly more than that of patients who answered "easy to use" for rebamipide. This result suggests that an eye drop with "easy-to-use" is favorable for dry eye therapy because the incidence of dry eye is especially high among older patients [2-5]. These results indicates that patient preferences varied from patient to patient, and understanding the practical characteristics of the eye drops is very important for proceeding with the treatment.

Clinical efficacy was also confirmed in this trial, generally satisfying with the previous evidences related to diquafosol and rebamipide in phase III studies $[21,29]$ and post-marketing surveillance [30]. These two new eye drops are also effective in clinical practice as a long time use, reported recently [31].
This study is the first to report patients' preferences with respect to two major, novel eye drops, diquafosol and rebamipide, in real-world dry eye patients based on a questionnaire. However, the present study has some limitations; (1) small number of enrolled patients, (2) single-center study design and short treatment period of a total 8 weeks, (3) bitterness information beforehand, and (4) no washout period. In the present study, the patients changed eye drops 4 times without washout period, because we placed importance on looking to the subjective usability of these eye drops and on continuing treatment with less emotional strain to them. No washout design may influence the results partway in this study. Further studies are needed to establish the patient preferences with respect to these eye drops.

In conclusions, diquafosol and rebamipide are similarly effective in treating dry eye subjectively and objectively in clinical practice, and there was no significant difference in the preference of them. Our survey also suggests that patient preferences vary depending on their individual conditions, bitterness from rebamipide and easiness in using the diquafosol bottle seemed to correlate with the trend of patient preferences with respect to these eye drops.

\section{Acknowledgements}

We thank Ms. Shino Katayama and Ms. Marina Tokoro for their technical assistance.

\section{References}

1. The definition and classification of dry eye disease: report of the Definition and Classification Subcommittee of the International Dry Eye WorkShop (2007). Ocul Surf. 2007 Apr;5(2):75-92.

2. The epidemiology of dry eye disease: report of the Epidemiology Subcommittee of the International Dry Eye WorkShop (2007). Ocul Surf. 2007 Apr;5(2):93107.

3. Connor CG, Flockencier LL, Hall CW. The influence of gender on the ocular surface. J Am Optom Assoc. 1999 Mar;70(3):182-6.

4. Moss SE, Klein R, Klein BE. Prevalence of and risk factors for dry eye syndrome. Arch Ophthalmol. 2000 Sep;118(9):1264-8.

5. Moss SE, Klein R, Klein BEK. Incidence of dry eye in an older population. Arch Ophthalmol. 2004 Mar;122(3):369-73.

6. Perry HD. Dry eye disease: pathophysiology, 
classification, and diagnosis. Am J Manag Care. 2008 Apr;14(3 Suppl):S79-87.

7. Nichols KK. Patient-reported symptoms in dry dye disease. Ocul Surf. 2006 Jul;4(3):137-45.

8. Miljanovic B, Dana R, Sullivan DA, Schaumberg DA. Impact of dry eye syndrome on vision-related quality of life. Am J Ophthalmol. 2007 Mar;143(3):409-15.

9. Goto E, Yagi Y, Matsumoto Y, Tsubota K. Impaired functional visual acuity of dry eye patients. Am J Ophthalmol. 2002 Feb;133(2):181-6.

10. Management and therapy of dry eye disease: report of the Management and Therapy Subcommittee of the International Dry Eye WorkShop (2007). Ocul Surf. 2007 Apr;5(2):163-78.

11. Friedman NJ. Impact of dry eye disease and treatment on quality of life. Curr Opin Ophthalmol. 2010 Jul;21(4):310-6.

12. Akpek EK, Smith RA. Current treatment strategies for age-related ocular conditions. Am J Manag Care. 2013 May;19(5 Suppl):S76-84.

13. Yokoi N, Bron A, Tiffany J, Brown N, Hsuan J, Fowler C. Reflective meniscometry: a non-invasive method to measure tear meniscus curvature. Br J Ophthalmol. 1999 Jan;83(1):92-7.

14. Shimmura S, Ono M, Shinozaki K, Toda I, Takamura E, Mashima $Y$, et al. Sodium hyaluronate eyedrops in the treatment of dry eyes. Br J Ophthalmol. 1995 Nov;79(11):1007-11.

15. Condon PI, McEwen CG, Wright M, Mackintosh G, Prescott RJ, McDonald C. Double blind, randomised, placebo controlled, crossover, multicentre study to determine the efficacy of a $0.1 \%(\mathrm{w} / \mathrm{v})$ sodium hyaluronate solution (Fermavisc) in the treatment of dry eye syndrome. $\mathrm{Br} J$ Ophthalmol. 1999 Oct;83(10):1121-4.

16. Aragona P, Di Stefano G, Ferreri F, Spinella R, Stilo A. Sodium hyaluronate eye drops of different osmolarity for the treatment of dry eye in Sjögren's syndrome patients. Br J Ophthalmol. 2002 Aug;86(8):879-84.

17. Lau OCF, Samarawickrama C, Skalicky SE. P2Y2 receptor agonists for the treatment of dry eye disease: a review. Clin Ophthalmol. 2014 Jan 30;8:327-34.

18. Kashima $T$, Itakura H, Akiyama H, Kishi S. Rebamipide ophthalmic suspension for the treatment of dry eye syndrome: a critical appraisal. Clin Ophthalmol. 2014 May 30;8:1003-10.

19. Nakamura M, Imanaka T, Sakamoto A. Diquafosol ophthalmic solution for dry eye treatment. Adv Ther.
2012 Jul;29(7):579-89.

20. Matsumoto $Y$, Ohashi $Y$, Watanabe H, Tsubota K, Diquafosol Ophthalmic Solution Phase 2 Study Group. Efficacy and safety of diquafosol ophthalmic solution in patients with dry eye syndrome: a Japanese phase 2 clinical trial. Ophthalmology. 2012 Oct;119(10):1954-60.

21. Takamura E, Tsubota K, Watanabe H, Ohashi Y, Diquafosol Ophthalmic Solution Phase 3 Study Group. A randomised, double-masked comparison study of diquafosol versus sodium hyaluronate ophthalmic solutions in dry eye patients. Br J Ophthalmol. 2012 Oct;96(10):1310-5.

22. Shigeyasu C, Yamada M, Akune Y, Tsubota K. Diquafosol sodium ophthalmic solution for the treatment of dry eye: clinical evaluation and biochemical analysis of tear composition. Jpn J Ophthalmol. 2015 Nov;59(6):415-20.

23. Shigeyasu C, Hirano S, Akune Y, Yamada M. Diquafosol Tetrasodium Increases the Concentration of Mucinlike Substances in Tears of Healthy Human Subjects. Current Eye Research. 2015 Sep 2;40(9):878-83.

24. Urashima $H$, Okamoto $T$, Takeji $Y$, Shinohara $H$, Fujisawa S. Rebamipide increases the amount of mucin-like substances on the conjunctiva and cornea in the $\mathrm{N}$-acetylcysteine-treated in vivo model. Cornea. 2004 Aug;23(6):613-9.

25. KASE S, SHINOHARA T, KASE M. Histological observation of goblet cells following topical rebamipide treatment of the human ocular surface: A case report. Exp Ther Med. 2015 Feb;9(2):456-8.

26. Shimazaki J. Definition and diagnosis of dry eye 2006. Atarashii Ganka. 2007 Jan 1;24:181-4.

27. Sakane $Y$, Yamaguchi M, Yokoi N, Uchino M, Dogru $M$, Oishi $T$, et al. Development and validation of the Dry Eye-Related Quality-of-Life Score questionnaire. JAMA Ophthalmol. 2013 Oct;131(10):1331-8.

28. Mah F, Milner M, Yiu S, Donnenfeld E, Conway TM, Hollander DA. PERSIST: Physician's Evaluation of Restasis(®) Satisfaction in Second Trial of topical cyclosporine ophthalmic emulsion $0.05 \%$ for dry eye: a retrospective review. Clin Ophthalmol. 2012;6:19716.

29. Kinoshita S, Oshiden K, Awamura S, Suzuki H, Nakamichi N, Yokoi N, et al. A randomized, multicenter phase 3 study comparing $2 \%$ rebamipide (OPC-12759) with $0.1 \%$ sodium hyaluronate in the treatment of dry eye. Ophthalmology. 2013 Jun;120(6):1158-65. 
30. Yamaguchi M, Nishijima T, Shimazaki J, Takamura E, Yokoi N, Watanabe $\mathrm{H}$, et al. Clinical usefulness of diquafosol for real-world dry eye patients: a prospective, open-label, non-interventional, observational study. Adv Ther. 2014 Nov;31(11):116981.

31. Yamane M, Ogawa Y, Fukui M, Kamoi M, Saijo-Ban Y, Yaguchi S, et al. Long-term rebamipide and diquafosol in two cases of immune-mediated dry eye. Optom Vis Sci. 2015 Apr;92(4 Suppl 1):S25-32.

Copyright: (c) Ohtani et al. This is an Open Access article distributed under the terms of the Creative Commons Attribution License, which permits unrestricted use, distribution, and reproduction in any medium, provided the original work is properly cited. 